\title{
Metabolic Characterization of Peripheral Host Responses to Drainage-Resistant Klebsiella pneumoniae Liver Abscesses by Serum 1H-NMR Spectroscopy
}

\author{
Zhihui Chang, Hairui Wang, Beibei Li, Zhaoyu Liu* and Jiahe Zheng* \\ Department of Radiology, Shengjing Hospital of China Medical University, Shenyang, China
}

Purpose: To explore the metabolic characterization of host responses to drainage-resistant Klebsiella pneumoniae liver abscesses (DRKPLAs) with serum $1 \mathrm{H}$-nuclear magnetic resonance (NMR) spectroscopy.

Materials and Methods: The hospital records of all patients with a diagnosis of a liver abscess between June 2015 and December 2016 were retrieved from an electronic hospital database. Eighty-six patients with Klebsiella pneumoniae (K. pneumoniae) liver abscesses who underwent percutaneous drainage were identified. Twenty patients with

OPEN ACCESS

Edited by:

David Dockrell,

University of Edinburgh

United Kingdom

Reviewed by:

Amit Kumar Mandal,

Raiganj University, India

Thomas C. Darton,

University of Sheffield,

United Kingdom

*Correspondence:

Zhaoyu Liu

liuzy1226@126.com

Jiahe Zheng

zhengjh1226@126.com

Received: 18 November 2017 Accepted: 07 May 2018

Published: 01 June 2018

Citation:

Chang Z, Wang H, Li B, Liu Z and

Zheng J (2018) Metabolic

Characterization of Peripheral Host

Responses to Drainage-Resistant

Klebsiella pneumoniae Liver

Abscesses by Serum 1H-NMR

Spectroscopy

Front. Cell. Infect. Microbiol. 8:174.

doi: 10.3389/fcimb.2018.00174 confirmed DRKPLAs were studied. Moreover, we identified 20 consecutive patients with drainage-sensitive Klebsiella pneumoniae liver abscesses (DSKPLAs) as controls. Serum samples from the two groups were analyzed with $1 \mathrm{H}$ NMR spectroscopy. Partial least squares discriminant analysis (PLS-DA) was used to perform $1 \mathrm{H}$ NMR metabolic profiling. Metabolites were identified using the Human Metabolome Database, and pathway analysis was performed with MetaboAnalyst 3.0.

Results: The PLS-DA test was able to discriminate between the two groups. Five key metabolites that contributed to their discrimination were identified. Glucose, lactate, and 3-hydroxybutyrate were found to be upregulated in DRKPLAs, whereas glutamine and alanine were downregulated compared with the DSKPLAs. Pathway analysis indicated that amino acid metabolisms were significantly different between the DRKPLAs and the DSKPLAs. The D-glutamine and D-glutamate metabolisms exhibited the greatest influences.

Conclusions: The five key metabolites identified in our study may be potential targets for guiding novel therapeutics of DRKPLAs and are worthy of additional investigation.

\footnotetext{
Keywords: metabolite, drainage-resistant, Klebsiella pneumoniae, liver abscess, $1 \mathrm{H}$-nuclear magnetic resonance spectroscopy
}

\section{INTRODUCTION}

Pyogenic liver abscess (PLA) is a life-threatening intra-abdominal infectious disease. In the two most recent decades, Klebsiella pneumoniae (K. pneumoniae) has become the most common causative pathogen of PLA in Asian countries as well as worldwide (Braiteh and Golden, 2007; Abate et al., 2012; Siu et al., 2012; Luo et al., 2014). 
The imaging features of $K$. pneumoniae liver abscesses (KPLAs) are similar to those of other types of liver abscesses. However, there are some notable differences. KPLAs more frequently appear as solid or multiloculated liver abscesses (Hui et al., 2007; Alsaif et al., 2011; Lee et al., 2011; Wang et al., 2014). Because of these features, some KPLAs are resistant to drainage because of the failure of liquefaction and require surgical intervention (Alkofer et al., 2012; Tan et al., 2013; Lo et al., 2015). Hence, it is of great importance to explore the host response mechanisms and develop treatment strategies to promote the liquefaction of KPLAs. However, to our knowledge, there are no reports that have explored the mechanism related to the failure of liquefaction of KPLAs. The failure of the treatment of a liver abscess by percutaneous drainage defines drainageresistant liver abscesses (De Jong et al., 2010). In this study, we focused on drainage-resistant Klebsiella pneumoniae liver abscesses (DRKPLAs), and they had the following features: (a) the maximum diameter of the abscess did not decrease 1 week after drainage, and the drainage tube position was located within the lesion (Figures 1A,B); (b) the patients still exhibited a high fever, chills and other symptoms, and the inflammation did not decrease as indicated, for example, by the failure of $\mathrm{C}$ reactive protein (CRP) levels or decrease in white blood cell counts.

Nuclear magnetic resonance (NMR)-based metabonomic analysis is a systems biology approach that aims to detect global metabolic information in biological samples. This approach is widely used in infectious disease diagnosis and the study of pathogenesis (Chen et al., 2014; Embade et al., 2016; Li et al., 2017).
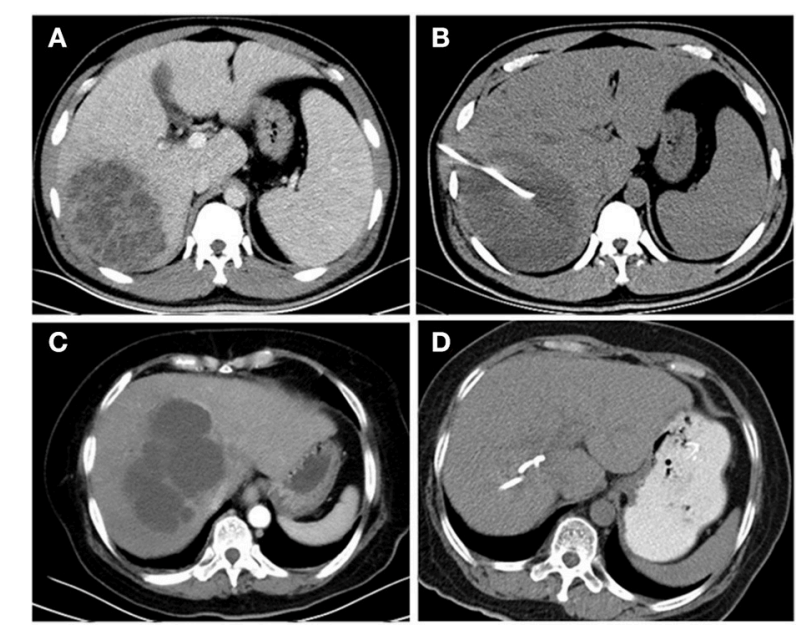

FIGURE 1 | CT images of the livers of $K$. pneumoniae liver abscess patients before and 1 week after drainage. A 36-year-old man with a K. pneumoniae liver abscess (A). The diameter of the abscess did not decrease 1 week after drainage (B), and the abscess was thus defined as a drainage-resistant $K$. pneumoniae liver abscess (DRKPLA). A 62-year-old woman with a $K$. pneumoniae liver abscess (C). The abscess had almost disappeared 1 week after drainage (D) and was thus defined as a drainage-sensitive $K$. pneumoniae liver abscess (DSKPLA).
Therefore, the purpose of this study was to retrospectively compare the metabolic characterizations of host responses to DRKPLAs and drainage-sensitive Klebsiella pneumoniae liver abscesses (DSKPLAs; Figures 1C,D) with serum 1H-NMR spectroscopy. Our results demonstrated that statistical analyses of serum $1 \mathrm{H}-\mathrm{NMR}$ spectra can effectively distinguish between these two groups of KPLA patients and thus provide a novel application of NMR spectroscopy.

\section{MATERIALS AND METHODS}

\section{Patients and Data}

All serum samples were collected prospectively in a previous study [Title:Predictors from Clinical and CT features for metastatic infection among Patients with Klebsiella pneumoniae Liver Abscess, Registration at the Chinese clinical trial center (http://www.chictr.org.cn) with number:ChiCTR-ROC15006581. The project was approved by the ethics committee of the Shengjing Hospital (Approved Number:2015PS184K), all patients provided written informed consent]. Shengjing hospital granted a waiver for this retrospective study.

Using our institutional electronic medical database, we retrieved the records of patients with a main diagnosis of a liver abscess between June 2015 and December 2016. The database search yielded 218 patients.

The inclusion criteria were as follows: (a) the presence of a focal lesion or lesions in the liver on contrast-enhanced CT images, (b) the receipt of percutaneous drainage therapy and the presence of frank pus aspirated from the abscess cavity through this procedure, and (c) positive microbiological culture

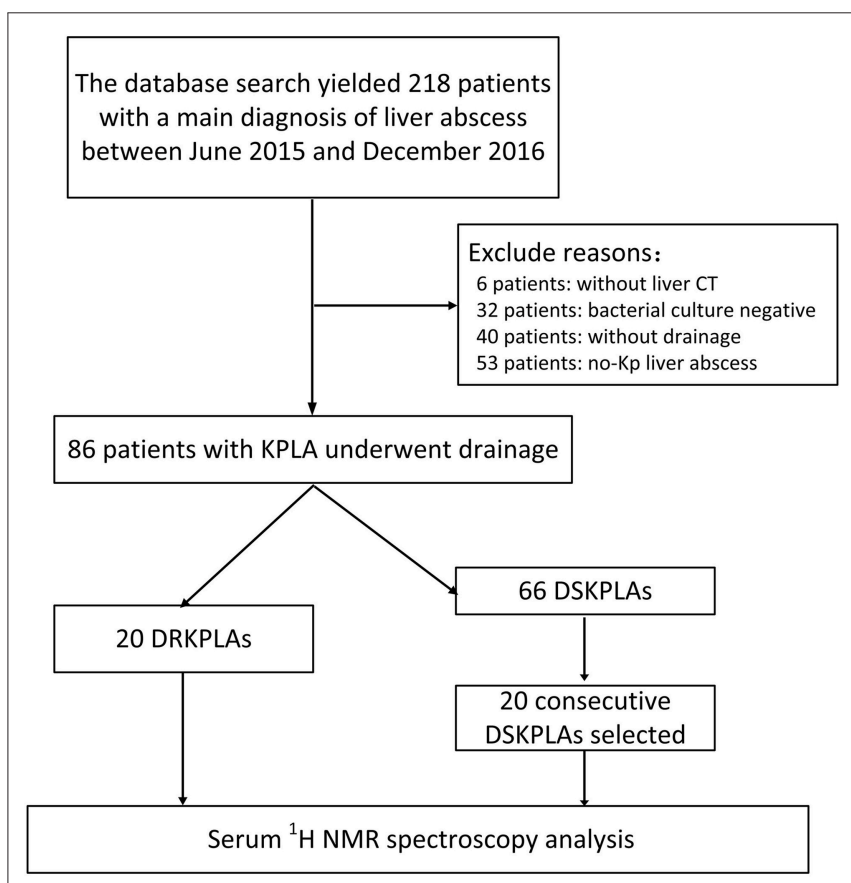

FIGURE 2 | Flow chart of the patient selection. 
TABLE 1 | Clinical and CT Characteristic in 86 patients with KPLA underwent drainage.

\begin{tabular}{|c|c|c|c|}
\hline Characteristic & DRKPLA $(n=20)$ & DSKPLA $(n=66)$ & $\boldsymbol{P}^{\star}$ \\
\hline Age (years) & $52.44 \pm 11.71$ & $55.83 \pm 10.97$ & 0.12 \\
\hline Sex (male) & $14(70.0)$ & $53(80.3)$ & 0.21 \\
\hline \multicolumn{4}{|l|}{ UNDERLYING DISEASE } \\
\hline Diabetes mellitus & $14(70.0)$ & $34(51.5)$ & 0.03 \\
\hline Cryptogenic & $5(25.0)$ & $17(25.7)$ & 0.26 \\
\hline Biliary tract disease & $1(5.0)$ & $6(9.1)$ & 0.32 \\
\hline \multicolumn{4}{|l|}{ PRESENTING SYMPTOMS } \\
\hline Fever $\left(\geq 38^{\circ} \mathrm{C}\right)$ & $19(95.0)$ & $60(90.9)$ & 0.68 \\
\hline Abdominal pain/discomfort & $17(85.0)$ & $51(77.2)$ & 0.43 \\
\hline Gastrointestinal symptoms & $4(20.0)$ & $18(27.3)$ & 0.11 \\
\hline \multicolumn{4}{|c|}{ HEMATOLOGICAL PARAMETERS } \\
\hline White blood cells $\left(\times 10^{9} / \mathrm{L}\right)$ & $16.03 \pm 5.59$ & $14.56 \pm 6.69$ & 0.31 \\
\hline $\mathrm{CRP}(\mathrm{mg} / \mathrm{L})$ & $183.91 \pm 143.74$ & $225.47 \pm 81.65$ & 0.51 \\
\hline ALT & $121.85 \pm 74.69$ & $90.60 \pm 51.97$ & 0.09 \\
\hline Total bilirubin & $28.25 \pm 13.20$ & $30.94 \pm 14.48$ & 0.19 \\
\hline Septic Shock & $2(10.0)$ & $5(7.6)$ & 0.30 \\
\hline Days of hospitalization & $21.76 \pm 12.38$ & $8.04 \pm 4.63$ & $<0.01$ \\
\hline \multicolumn{4}{|l|}{ CT CHARACTERISTIC } \\
\hline $\begin{array}{l}\text { Maximal abscess } \\
\text { diameter(mm) }\end{array}$ & $62.87 \pm 29.77$ & $69.67 \pm 34.58$ & 0.06 \\
\hline Single & $16(80.0)$ & $44(66.7)$ & 0.10 \\
\hline Solid & $19(95.0)$ & $37(56.1)$ & 0.02 \\
\hline Multilocular & $16(80.0)$ & $38(57.6)$ & 0.04 \\
\hline Gas formation & $1(5.0)$ & $14(21.2)$ & $<0.01$ \\
\hline $\begin{array}{l}\text { Hypermucoviscosity } \\
\text { phenotype }\end{array}$ & $17(85.0)$ & $31(46.9)$ & 0.01 \\
\hline
\end{tabular}

Data are presented as median (mean \pm standard deviation) or $n$ (\%). DRKPLA, drainageresistant Klebsiella pneumoniae liver abscess; DSKPLA, drainage-sensitive Klebsiella pneumoniae liver abscesses. ${ }^{*}$ DRKPLA vs. DSKPLA.

results from the liver abscess and/or blood cultures in addition to culture results that indicated the presence of mono-microbial K. pneumoniae.

The medical records were reviewed for the following information: (a) demographic data; (b) coexisting medical conditions, including diabetes mellitus, biliary tract disease, malignancy, and cirrhosis; (c) clinical symptoms; (d) initial laboratory data, including the total and differential white blood cell counts, platelet counts, glucose, C-reactive protein and immune function (lymphocyte subsets); and (e) whether the patients underwent a surgical intervention.

\section{Lymphocyte Subsets by Flow Cytometry}

Flow cytometric enumeration of $\mathrm{T}, \mathrm{B}$, and NK cells were performed on FACSCalibur (BD Biosciences, San Jose, CA, USA) and the different cell populations were analyzed using the Cell Quest Pro software (v5.2). The monoclonal antibodies used to identify these cell subsets were as follows: anti-CD45, anti-CD3, anti-CD4, anti-CD8, anti-CD19, anti-CD16, anti-CD56, anti-CD45RA, and anti-CD45RO. Briefly, whole blood $(100 \mu \mathrm{L})$ was labeled with respective
TABLE 2 | Analysis of peripheral blood lymphocyte subsets in seven patients.

\begin{tabular}{|c|c|c|c|}
\hline \multirow[t]{2}{*}{ Items } & \multicolumn{2}{|c|}{ The proportions of lymphocyte subsets $\%$} & \multirow[t]{2}{*}{$\boldsymbol{p}^{\star}$} \\
\hline & $\operatorname{DRKPLA}(n=3)$ & $\operatorname{DSKPLA}(n=4)$ & \\
\hline \multicolumn{4}{|c|}{ T CELL SURFACE ANTIGENS } \\
\hline CD3+ & $85.04 \pm 5.84$ & $84.22 \pm 2.42$ & 0.81 \\
\hline $\mathrm{CD} 3+\mathrm{CD} 8+$ & $39.89 \pm 16.00$ & $33.78 \pm 20.66$ & 0.69 \\
\hline $\mathrm{CD} 3+\mathrm{CD} 4+$ & $33.76 \pm 27.58$ & $48.62 \pm 19.12$ & 0.43 \\
\hline $\mathrm{CD} 4+\mathrm{CD} 45 \mathrm{RA}+$ & $29.08 \pm 7.14$ & $52.75 \pm 28.00$ & 0.22 \\
\hline $\mathrm{CD} 4+\mathrm{CD} 45 \mathrm{RO}+$ & $19.33 \pm 1.32$ & $48.12 \pm 20.14$ & 0.06 \\
\hline \multicolumn{4}{|c|}{ B CELL SURFACE ANTIGENS } \\
\hline CD19+ & $8.77 \pm 6.72$ & $6.45 \pm 3.74$ & 0.58 \\
\hline \multicolumn{4}{|c|}{ NK CELL SURFACE ANTIGENS } \\
\hline CD16+CD56+ & $4.76 \pm 1.20$ & $8.12 \pm 1.21$ & 0.02 \\
\hline
\end{tabular}

Data are presented as median (mean \pm standard deviation). DRKPLA, drainage-resistant Klebsiella pneumoniae liver abscess; DSKPLA, drainage-sensitive Klebsiella pneumoniae liver abscesses. ${ }^{*} D R K P L A$ vs. DSKPLA.

TABLE 3 | Characteristics of the serum sample population.

\begin{tabular}{lccc}
\hline Characteristic & DRKPLA $(\boldsymbol{n}=\mathbf{2 0})$ & DSKPLA $(\boldsymbol{n}=\mathbf{2 0})$ & $\boldsymbol{P}^{\boldsymbol{*}}$ \\
\hline Age (years) & $52.44 \pm 11.71$ & $54.63 \pm 11.29$ & 0.15 \\
Sex (male) & $14(70.0)$ & $15(75.0)$ & 0.28 \\
BMI & $23.49 \pm 2.49$ & $22.3 \pm 2.17$ & 0.36 \\
COMBINED DISEASE & & & \\
Diabetes mellitus & $14(70.0)$ & $9(45.0)$ & 0.01 \\
Hypertension & $5(25.0)$ & $4(20.0)$ & 0.62 \\
Hyperlipemia & $6(30.0)$ & $7(35.0)$ & 0.52 \\
Liver cirrhosis & $1(0.025)$ & 0 & 0.29 \\
\hline
\end{tabular}

Data are presented as median (mean \pm standard deviation) or $n$ (\%). DRKPLA, drainageresistant Klebsiella pneumoniae liver abscess; DSKPLA, drainage-sensitive Klebsiella pneumoniae liver abscesses. *DRKPLA vs. DSKPLA.

antibodies (BD biosciences, USA) as per manufacturer's instructions. After $30 \mathrm{~min}$ incubation in dark at room temperature with mAbs, RBC's were lysed using FACS lysing solution and washed with phosphate buffered saline. Labeled lymphocytes were acquired on a FACSCalibur flow cytometer (BD Biosciences, San Jose, CA, USA) after proper instrument setting, calibration, and compensation. Absolute counts (Abs) for each cell subset were calculated by multiplying the specific subset percentages to absolute lymphocyte counts/100. The lymphocytes were gated as $T$ cells (CD3+, CD3+CD8+, CD3+CD4+, CD4+CD45RA+, and $\mathrm{CD} 4+\mathrm{CD} 45 \mathrm{RO}+)$, B cells $(\mathrm{CD} 19+)$, and $\mathrm{NK}$ cells $(\mathrm{CD} 16+\mathrm{CD} 56+)$.

\section{K. pneumoniae String Test}

The hypermucoviscous phenotype of $K$. pneumoniae can be identified by a positive string test. A bacterial loop was touched to a suspect colony on an agar plate and withdrawn slowly, and the bacteria that formed a mucoid "string" of $5 \mathrm{~mm}$ were considered to be positive (Wang et al., 2013). 


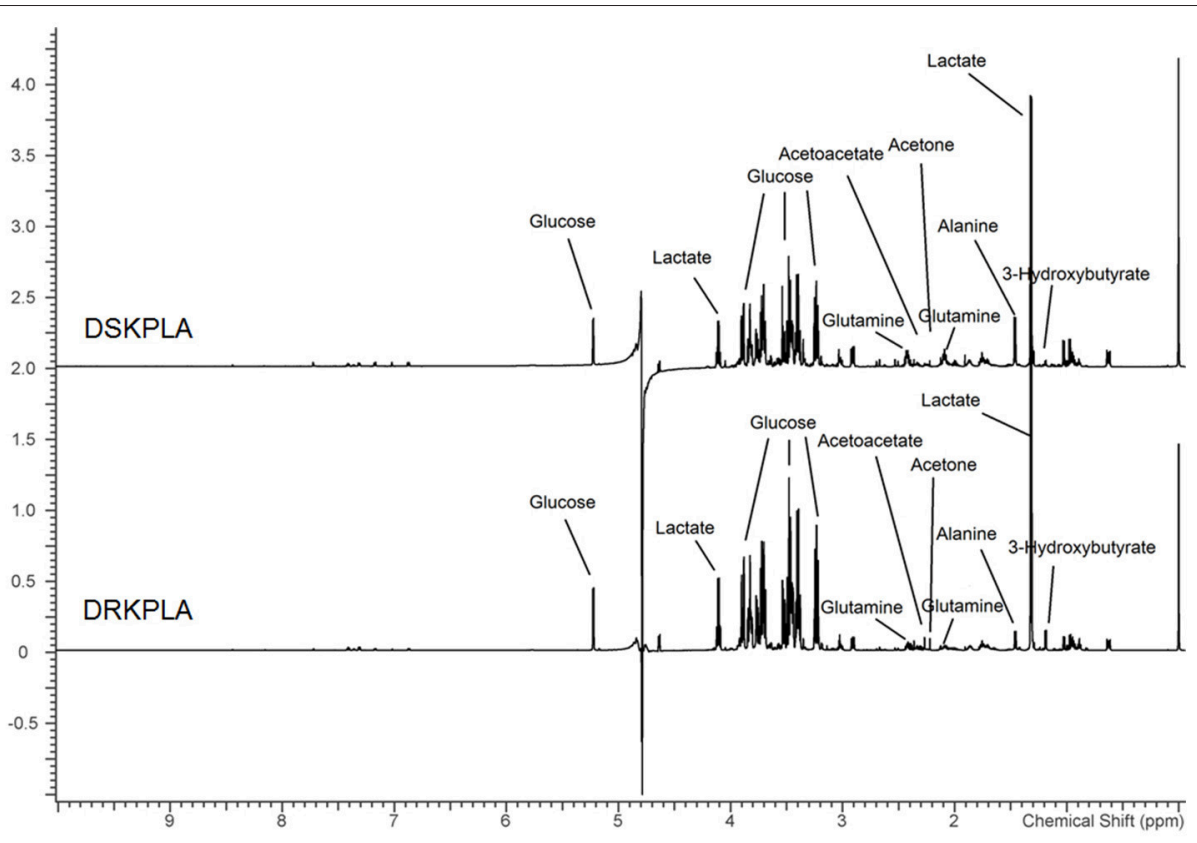

FIGURE 3 | Representative 599.83 MHz 1HNMR spectra of serum samples from a DSKPLA and a DRKPLA patient. The key metabolites are noted.

\section{CT Characteristics}

All patients underwent contrast-enhanced CT prior to the drainage of the liver abscesses. If the clinical symptoms were not alleviated 3 days after drainage, or the quantity of drainage was very small, liver CT studies were also performed to assess the size of the abscess cavity and to determine the position of the drainage catheter in cases of poor or incomplete drainage or to monitor complications. Both CT examinations were performed, and the scans were reviewed as previously reported (Chang et al., 2015, 2016).

\section{Treatment}

In addition to the percutaneous drainage therapy, all patients received antibiotic therapy (most commonly third-generation cephalosporins, ciprofloxacin, or carbapenem antibiotics) for their abscesses.

\section{${ }^{1} \mathrm{H}-\mathrm{NMR}$ Spectra}

For the $1 \mathrm{H}$-NMR analyses, $500 \mu \mathrm{L}$ serum samples were thawed at a temperature of $4^{\circ} \mathrm{C}$ and then passed through a $3-\mathrm{KDa}$ ultrafiltration filter (Millipore, USA). The filtered units were centrifuged at 13,000 rpm for $30 \mathrm{~min}$ at $4^{\circ} \mathrm{C}$. The filtrates were collected and mixed with $50 \mu \mathrm{L}$ Anachro Certified DSS Standard Solution (ACDSS), vortexed (10 s) and centrifuged (13,000 rpm, 2 min, $4^{\circ} \mathrm{C}$ ). The prepared NMR samples were loaded into 5 $\mathrm{mm}$ NMR tubes. The $1 \mathrm{H}$-NMR measurements were performed at $298.15 \mathrm{~K}$ on an Agilent $600 \mathrm{MHz}$ spectrometer equipped with a triple-resonance cryoprobe (Agilent Technologies, USA) that was operated at $599.83 \mathrm{MHz}$ to examine the $1 \mathrm{H}$ resonance frequency. A METNOESYpulse sequence was used to determine the serum metabolite profiles (Weljie et al., 2006). Sixty-four transitions were collected with a frequency domain size of $65,536 \mathrm{~Hz}$. The relaxation delay time was set to $1 \mathrm{~s}$, which matched the spectra acquisition condition of the Chenomx reference standard database. The $1 \mathrm{H}-\mathrm{NMR}$ free induction decay signal was imported into the Chenomx NMR suite version 8.0 (Chenomx, Canada), and the data were automatically Fourier transformed and phaseadjusted. The baseline was carefully adjusted by experienced technicians inside the processor module. Metabolites from the serum were qualified and quantified using the Chenomx NMR Suite software package (Tredwell et al., 2011). Briefly, the qualification was performed by matching the sample spectra against the spectra of a $\mathrm{pH}$-adjusted standard reference inside the Chenomx Database. The quantification was performed by comparing the area under the deconvoluted peak against the peak area of the 2,2-dimethyl-2-silapentane-5-sulfonate sodium salt (DSS) signal at $0 \mathrm{ppm}$.

Partial least square discriminant analysis (PLS-DA) was applied to analyze the spectral data and separate the DRKPLA from the DSKPLA samples. Loading plots were used to identify the spectral variables that were responsible for sample separation in the corresponding score plots. The variable importances in the projections (VIPs) of all peaks from the PLS-DA models were taken as the coefficients for metabolite selection, and the variables with VIP values $>1$ were considered to be contributors to the group discrimination (Afanador et al., 2014). Student's $t$-test $(p<0.05)$ was also used to detect significant differences in metabolites between the DRKPLA and DSKPLA samples using Metaboanalyst ver 3.0 (http:// www.metaboanalyst.ca/MetaboAnalyst/). Only the variables with VIP values $>1$ and $p$ values $<0.05$ were considered significant. 

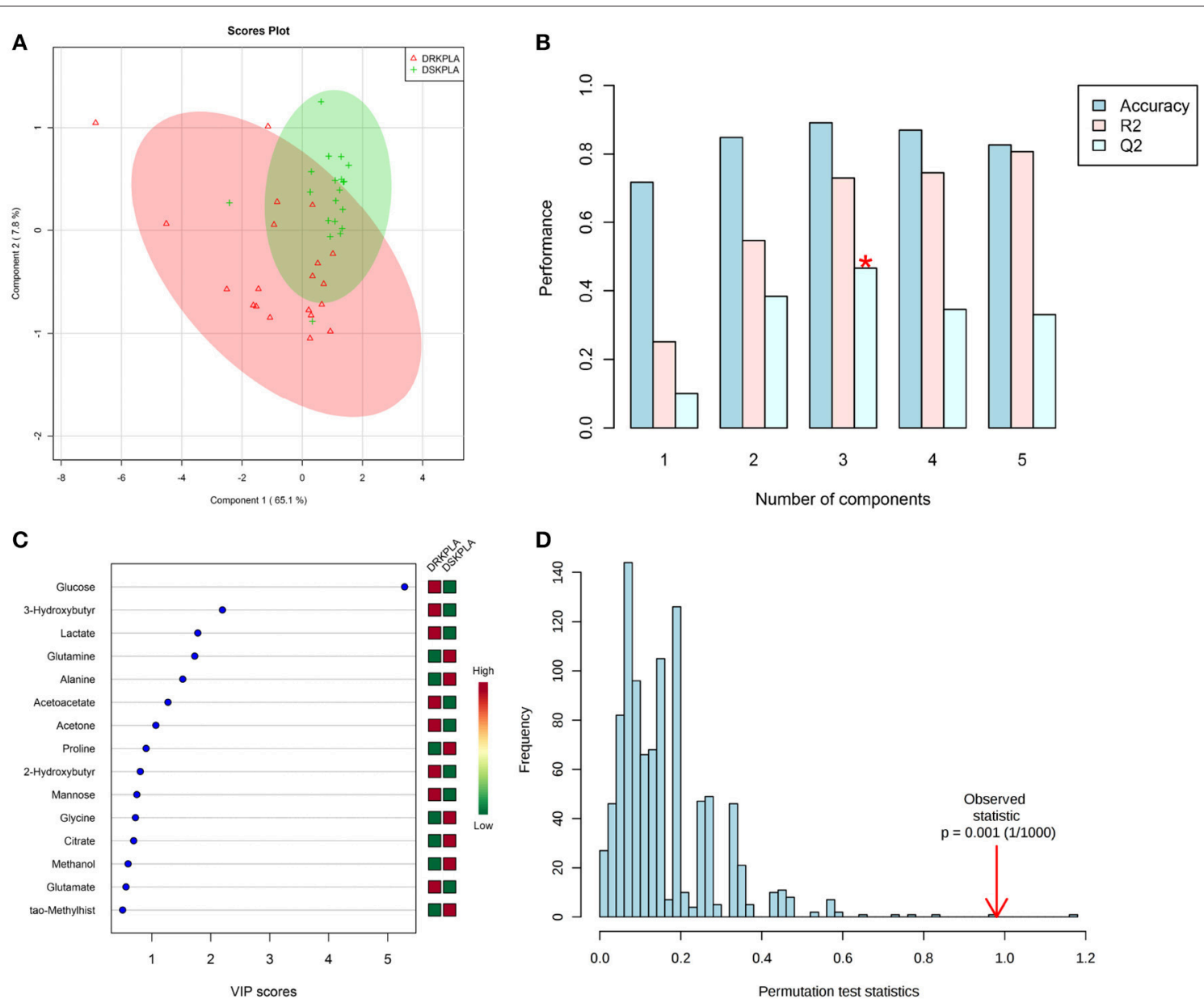

FIGURE 4 | PLS-DA analyses of the DRKPLAs and the DSKPLAs serum samples. (A) Two-dimensional PLS-DA score plot. (B) PLS-DA classification using different numbers of components. The red asterisk indicates the best classifier. The inset table summarizes the $Q^{2}, R^{2}$ and accuracy of the best model. (C) Important features identified by the VIP scores. The variable importance in projection score is a weighted sum of squares of the PLS-DA loadings that accounts for the amount of explained Y-variation in each dimension. (D) Permutation test statistics for 1,000 permutations with the observed statistic at $p<0.01$.

\section{Pathway Analysis}

Metaboanalyst version 3.0 was used to perform the pathway analysis and to visualize all of the chemical metabolites that were present at different levels in the serum samples from the DSKPLA and DRKPLA patients (Xia et al., 2015).

\section{Statistical Analysis}

Data about the subjects were analyzed using SPSS, version 17.0 (SPSS Inc.). Normally distributed continuous variables are presented as the means \pm the SDs and were compared using Student's $t$-test. Categorical variables were compared using Fisher's exact test. All tests were two-sided, and $p<0.05$ was considered statistically significant.

\section{RESULTS}

\section{Clinical Features}

In total, 86 patients with $K$. pneumoniae liver abscesses who underwent percutaneous drainage were identified. The patient selection process is illustrated in in Figure 2. Twenty patients (23.3\%) had DRKPLAs, and 66 patients (76.7\%) had DSKPLAs. Comparisons of the demographic data, laboratory findings, and other clinical features of the patients with DRKPLAs and DSKPLAs are presented in Table 1. The DRKPLA group has a significantly higher rate of diabetes compare to DSKPLA group $(70 \%$ vs. $51.5, p=0.03)$. The $K$. pneumoniae strain causing infection in the DRKPLA group is more likely to be of the hyperviscous phenotype ( 80 vs. $46.9 \%, p=0.01$ ). The days of hospitalization in DRKPLA group is significantly longer than DSKPLA group $(21.76 \pm 12.38$ vs. $8.04 \pm 4.63$ days, $p<0.01)$.

\section{CT Characteristics}

Comparisons of the CT characteristics of the DRKPLAs (Figures 1A,B) with the DSKPLAs (Figures 1C,D) are summarized in Table $\mathbf{1}$. The DSKPLAs predominantly exhibited solid CT appearances, were multilocular, and exhibited less gas formation $(p<0.05)$. 

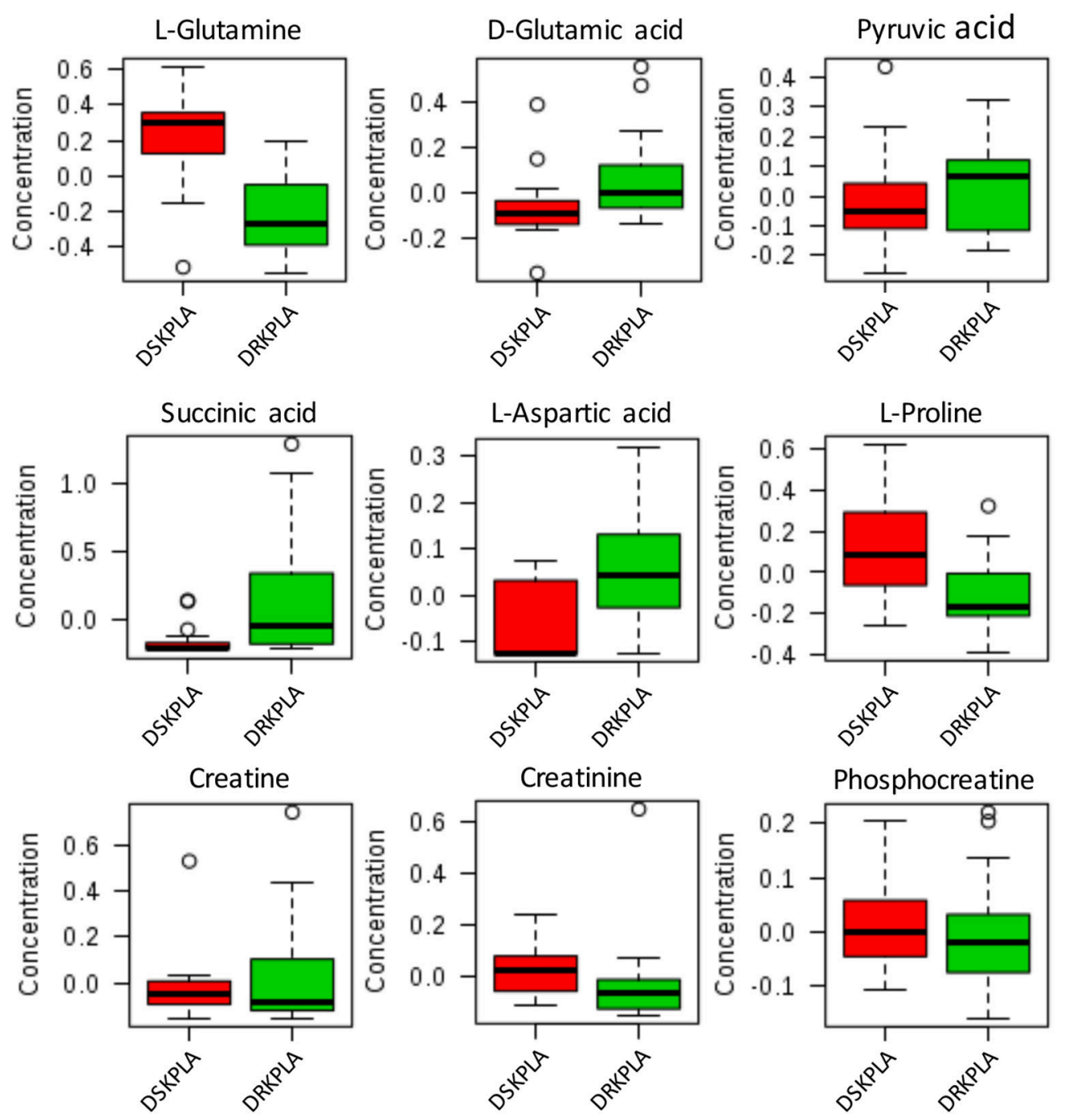

FIGURE 5 | Boxplot of the relative concentrations of the significantly altered metabolites ( $p<0.05)$ in the sera of DRKPLA (green) and DSKPLA (red) patients. The bar plots show the normalized values (the mean \pm one standard deviation). The boxes range from the $25 \%$ to the $75 \%$ percentiles, and the $5 \%$ and $95 \%$ percentiles are indicated as error bars. Single data points are indicated by circles. The medians are indicated by the horizontal lines within each box.

\section{Immune Function Analysis}

Seven patients underwent immune function tests in this study. The CD16+CD56+NK cells were significantly decreased in the DRKPLA patients compared with the DSKPLA patients $(p=0.02)$. The analyses of the peripheral blood lymphocyte subsets are presented in Table 2.

\section{Characteristics of the Serum Sample Population}

We collected a total of 40 serum samples, including 20 DRKPLAS and 20 DSKPLAs (to reduce costs, we only selected 20 consecutive patients as the controls), which were included in the metabolomics study. The demographic information about these patients is summarized in Table 3. The DRKPLA group has only a higher rate of diabetes compare to DSKPLA group (70 vs. $45 \%$, $p=0.01)$ in the study of serum metabolomics. Other consistent characteristics reduce the influences of differences in individual conditions and ensure that the findings are attributable to the two disease conditions.

\section{Analysis of the Serum ${ }^{1} \mathrm{H}$ NMR Spectra Reveals Distinct Metabolic Features}

A series of changes in the endogenous metabolite levels was observed when the DRKPLA patients were compared with the DSKPLA patients. Typical spectra of the serum samples from a DRKPLA patient and a DSKPLA patient are presented in Figure 3. We used PLS-DA to visualize the metabolic differences between these two patients. There were separate trends in both groups in the PLS-DA score plots (Figure 4A), which indicated that they had different metabolic characteristics. To evaluate the statistical robustness of the analysis, a 10-fold cross-validation was performed, and the $Q^{2}$ and $R^{2}$ values were deduced. Our 


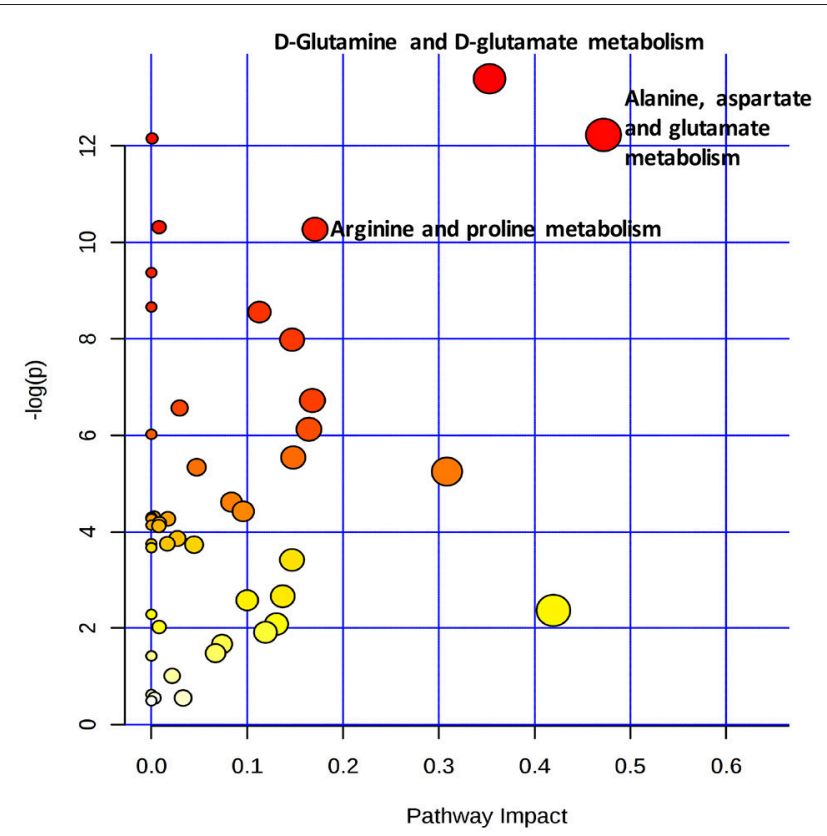

FIGURE 6 | Metabolic pathway analysis of the key metabolites present in the serum samples. The pathways named in the figure had impact values $>0.15$ and $-\log (p)$ values $>10$

model provided high $R^{2}$ and $Q^{2}$ scores, which confirmed a good predictive power (Figure 4B). Figure 4C illustrates the key differentiating features that were identified by the PLS-DA analysis sorted by increasing VIP score. Permutation tests $(1,000$ repeats) yielded a very low $p$-value $(<0.01)$, which indicated that none of the distributions formed by the permuted data were better than the observed statistic based on the original data (Figure 4D).

Five metabolites that could be used to discriminate between DRKPLAs and DsKPLAs were identified based on chemical shifts (VIP $>1$ and $p<0.05$ ). Glucose, lactate and 3-hydroxybutyrate were found to be upregulated in the DRKPLAs, and glutamine and alanine were downregulated in the DRKPLAs compared with the DSKPLAs. Figure 5 provides some example boxplots of metabolites that were significantly different in the serum of the DRKPLAs and DSKPLAs patients. All metabolites concentration is provided in Supplementary Table 1.

\section{Pathway Analysis}

With A combination of an pathway impact $>0.15$ and $-\log (\mathrm{p})$ $>10$, the results of the pathway analysis revealed that the three most strongly influence canonical pathways were alanine, aspartate and glutamate metabolism, D-glutamine and Dglutamate metabolism, and arginine and proline metabolism (Figure 6 and Table 4).

\section{DISCUSSION}

In addition to effective antimicrobial therapy, percutaneous drainage is recognized as the most safe and effective treatment for PLA because this treatment can reduce the pressure inside the abscess and lower the bacterial load (Liu et al., 2009; Liao et al., 2012). The effectiveness of drainage depends on the extent of liquefaction of the abscess and whether it is multilocular (Hui et al., 2007; Liao et al., 2012). Therefore, it is much more difficult to drain KPLAs that are characterized as solid or multiloculated. The data from the present study further strengthen the evidence that KPLAs with solid or multilocular appearances are associated with difficult drainage.

Our study results revealed that the hypermucoviscous strain of $K$. pneumoniae may be one of the causes of drainage resistance. Sticky pus can block the drainage tube or slow down the process of drainage. The high level of virulence observed in hypervirulent $K$. pneumoniae strains is due to an increased expression of capsular material that is related to the hypermucoviscous phenotype (Keynan et al., 2007; Wang et al., 2013). The factor that mediates the expression of the hypermucoviscous phenotype is RmpA/RmpA2 (Chuang et al., 2013; Qu et al., 2015). Because we don't have microbiology experiment conditions, we could not detect the serum types and virulence genes of $K$. pneumoniae. The other possible explanation for the solid appearance of KPLAs is the failure of liquefaction due to a high prevalence of a phagocytosisresistant capsular serotype K. pneumoniae strain (Hui et al., 2007; Alsaif et al., 2011). However, thus far, there is no experimental evidence to support this notion. However, we found that CD16+CD56+ natural killer (NK) cells were significantly decreased in the DRKPL compared with the DSKPLAs via an analysis of the peripheral blood lymphocyte subsets. NK cells are innate lymphocytes that play important roles in the defense against microbial pathogens through the secretion of IFN- $\gamma$ and recognition and lysis of virally or bacterially infected host cells (Fuchs and Colonna, 2011). On the other hand, diabetes can weaken the phagocytosis of macrophage and impaired host immunity. Both the engulfing and digesting of bacteria by host cells and the lysis of bacterial-infected host cell are associated with the liquefaction of abscesses.

To further explore the host response mechanisms, we used ${ }^{1} \mathrm{H}$ NMR-based metabonomic analysis with the aim of detecting global metabolic information from the sera of DRKPLA patients and comparing the results to those from DSKPLA patients. We found that the discrimination between the two patient groups was indeed possible via comparisons of the concentrations of metabolites. Glucose was found to be upregulated in DRKPLAs. Patients with diabetes are at an increased risk of KPLA due to impaired host immunity, poor blood supply, nerve damage, and alternations in metabolism (Wang et al., 2014). Thus, strict control of glucose during the treatment of an abscess is very important. In addition to glucose, four key metabolites identified in our study may be potential targets for promoting the liquidation of DRKPLAs, i.e., lactate, 3-hydroxybutyrate, glutamine and alanine. However, there is little information available from related studies with which to compare this finding. Chen et al. found different metabolic profiles of K1 serotype and non-serotype $\mathrm{K} 1$ and $\mathrm{K} 2$ Klebsiella pneumoniae isolates in a mouse model of oral infection, but these authors did not study the relationships of the serotype with abscess 
TABLE 4 | Pathway analysis of key metabolites.

\begin{tabular}{|c|c|c|c|c|c|}
\hline Pathway name & Hits $^{a}$ & $p$ & $-\log (p)$ & FDR $^{b}$ & Impact $^{c}$ \\
\hline Alanine, aspartate and glutamate metabolism & 2 & 1.53E-06 & 13.392 & 7.49E-05 & 0.35294 \\
\hline D-Glutamine and D-glutamate metabolism & 4 & 4.89E-06 & 12.228 & 8.61E-05 & 0.47199 \\
\hline Arginine and proline metabolism & 8 & 3.46E-05 & 10.271 & 3.39E-04 & 0.17075 \\
\hline Butanoate metabolism & 4 & 0.003915 & 5.543 & 0.011989 & 0.148 \\
\hline Purine metabolism & 3 & 3.31E-05 & 10.317 & 3.39E-04 & 0.00794 \\
\hline Nitrogen metabolism & 6 & 5.27E-06 & 12.154 & 8.61E-05 & 6.70E-04 \\
\hline Aminoacyl-tRNA biosynthesis & 10 & $1.92 E-04$ & 8.5565 & 0.001178 & 0.11268 \\
\hline Glyoxylate and dicarboxylate metabolism & 4 & $3.41 E-04$ & 7.9839 & 0.001856 & 0.14685 \\
\hline Citrate cycle (TCA cycle) & 3 & 0.001201 & 6.725 & 0.005883 & 0.005883 \\
\hline Fructose and mannose metabolism & 1 & 0.001404 & 6.5682 & 0.006256 & 0.02948 \\
\hline Methane metabolism & 5 & 0.002181 & 6.128 & 0.008475 & 0.16439 \\
\hline Synthesis and degradation of ketone bodies & 3 & 0.003696 & 5.6006 & 0.011989 & 0.7 \\
\hline Tyrosine metabolism & 4 & 0.004791 & 5.341 & 0.01381 & 0.01381 \\
\hline Glycine, serine, and threonine metabolism & 7 & 0.005242 & 5.2511 & 0.014269 & 0.30843 \\
\hline Valine, leucine, and isoleucine degradation & 5 & 0.009874 & 4.6178 & 0.025465 & 0.0835 \\
\hline Glycolysis or Gluconeogenesis & 5 & 0.011955 & 4.4266 & 0.028519 & 0.09576 \\
\hline Galactose metabolism & 3 & 0.013393 & 4.313 & 0.028519 & 0.00276 \\
\hline Starch and sucrose metabolism & 1 & 0.013969 & 4.2709 & 0.028519 & 0.01703 \\
\hline Primary bile acid biosynthesis & 1 & 0.015259 & 4.1826 & 0.029381 & 0.00822 \\
\hline Glutathione metabolism & 2 & 0.01619 & 4.1234 & 0.029381 & 0.00762 \\
\hline Vitamin B6 metabolism & 2 & 0.020978 & 3.8643 & 0.036712 & 0.02712 \\
\hline Cysteine and methionine metabolism & 2 & 0.023407 & 3.7547 & 0.037672 & 0.01649 \\
\hline Propanoate metabolism & 6 & 0.023833 & 3.7367 & 0.037672 & 0.04451 \\
\hline Lysine degradation & 3 & 0.032891 & 3.4146 & 0.048838 & 0.14675 \\
\hline Inositol phosphate metabolism & 1 & 0.070177 & 2.6567 & 0.10114 & 0.13703 \\
\hline Lysine biosynthesis & 2 & 0.076268 & 0.076268 & 0.10677 & 0.09993 \\
\hline Pyruvate metabolism & 4 & 0.094084 & 2.3636 & 0.12806 & 0.41957 \\
\hline Ascorbate and aldarate metabolism & 3 & 0.12483 & 2.0808 & 0.16097 & 0.13047 \\
\hline Phenylalanine metabolism & 4 & 0.14801 & 1.9105 & 0.18131 & 0.11906 \\
\hline Valine, leucine, and isoleucine biosynthesis & 5 & 0.18881 & 1.667 & 0.22565 & 0.07367 \\
\hline Glycerophospholipid metabolism & 2 & 0.22803 & 1.4783 & 0.26604 & 0.06691 \\
\hline Taurine and hypotaurine metabolism & 2 & 0.36489 & 1.0082 & 0.39733 & 0.02158 \\
\hline Selenoamino acid metabolism & 1 & 0.57638 & 0.55098 & 0.58839 & 0.00321 \\
\hline
\end{tabular}

${ }^{a}$ Hits, the number of compounds that match with our experimental data; ${ }^{b} \mathrm{FDR}$, False Discovery Rate; ${ }^{c}$ Impact, pathway impact value calculated from pathway topology analysis.

liquefaction or the prognosis of puncture drainage (Chen et al., 2014). The K1/K2 capsular polysaccharide (CPS) has proven to be significantly more resistant to phagocytosis than the non$\mathrm{K} 1 / \mathrm{K} 2 \mathrm{CPS}$ in liver abscess isolates (Lin et al., 2004; Yeh et al., 2006). The pathway analysis in our study revealed that alanine, aspartate and glutamate metabolism, D-glutamine and D-glutamate metabolism, and arginine and proline metabolism were the three most influential canonical KEGG pathways, which suggests that there is are significant differences in the amino acid profiles of sera from DRKPLA and DSKPLA patients. Dietary glutamine supplementation could partly reverse the impaired macrophage function and could increase NK cell activity and reduce the immunosuppressive effects that result from overload training (Song et al., 2015; Xiao et al., 2015). Therefore, we hypothesized that supplementation with glutamine may improve macrophage phagocytosis and the host immune defense against K. pneumoniae and thus promote abscess liquefaction. Animal experiments are expected to verify our hypothesis in the future.

Our study had limitations. First, it was retrospective in design. Second, in the serum metabolic analysis, only 20 cases were included as controls, which might have led to potential bias. Third, because the serum samples were obtained after the patients were diagnosed with liver abscesses, we cannot entirely rule out the notion that drainage resistance led to increased inflammation and then affected the metabolic changes. However, regardless of causality, the goal of this study was simply to identify potential targets for promoting the liquefaction of DRKPLAs.

In conclusion, our study results demonstrate that glucose and the other four key identified metabolites may be potential targets 
for guiding novel therapeutics of DRKPLAs and are worthy of additional investigation.

\section{AUTHOR CONTRIBUTIONS}

ZC, HW, BL, and ZL designed and conduct the study, analyzed the data, and draft the manuscript. JZ and ZL supervised the study. All authors read and approved the final manuscript.

\section{REFERENCES}

Abate, G., Koh, T. H., Gardner, M., and Siu, L. K. (2012). Clinical and bacteriological characteristics of Klebsiella pneumoniae causing liver abscess with less frequently observed multi-locus sequences type, ST163, from Singapore and Missouri, US. J. Microbiol. Immunol. Infect. 45, 31-36. doi: 10.1016/j.jmii.2011.09.002

Afanador, N. L., Tran, T. N., Blanchet, L., and Buydens, L. M. C. (2014). Variable importance in PLS in the presence of autocorrelated data-Case studies in manufacturing processes. Chemometr. Intell. Lab. Syst. 139, 139-145. doi: 10.1016/j.chemolab.2014.09.008

Alkofer, B., Dufay, C., Parienti, J. J., Lepennec, V., Dargere, S., and Chiche, L. (2012). Are pyogenic liver abscesses still a surgical concern? A Western experience. HPB Surg. 2012:316013. doi: 10.1155/2012/3 16013

Alsaif, H. S., Venkatesh, S. K., Chan, D. S., and Archuleta, S. (2011). CT appearance of pyogenic liver abscesses caused by Klebsiella pneumoniae. Radiology 260, 129-138. doi: 10.1148/radiol.11101876

Braiteh, F., and Golden, M. P. (2007). Cryptogenic invasive Klebsiella pneumoniae liver abscess syndrome. Int. J. Infect. Dis. 11, 16-22. doi: 10.1016/j.ijid.2005.10.006

Chang, Z., Gong, Z., Zheng, J., Ma, Y., and Liu, Z. (2016). Computed tomography features of septic pulmonary embolism caused by Klebsiella pneumoniae liver abscess associated with extrapulmonary metastatic infection. J. Comput. Assist. Tomogr. 40, 364-369. doi: 10.1097/RCT.00000000000 00383

Chang, Z., Zheng, J., Ma, Y., and Liu, Z. (2015). Analysis of clinical and CT characteristics of patients with Klebsiella pneumoniae liver abscesses: an insight into risk factors of metastatic infection. Int. J. Infect. Dis. 33, 50-54. doi: 10.1016/j.ijid.2014.12.041

Chen, N., Wang, L. L., Xue, J., Ma, X. B., Zhao, S., Rong, R. X., et al. (2014). Different metabolic profiles of K1 serotype and non-serotype K1 and K2 Klebsiella pneumoniae isolates in oral infection mice model. Microb. Pathog. 75, 41-48. doi: 10.1016/j.micpath.2014.08.006

Chuang, Y. C., Lee, M. F., Tan, C. K., Ko, W. C., Wang, F. D., and Yu, W. L. (2013). Can the rmpA gene predict metastatic meningitis among patients with primary Klebsiella pneumoniae liver abscess? J. Infect. 67, 166-168. doi: 10.1016/j.jinf.2013.03.016

De Jong, K. P., Prins, T. R., and Hofker, H. S. (2010). A new interventional technique for percutaneous treatment of drainage-resistant liver abscess. $\mathrm{Br}$. J. Radiol. 83, e195-e197. doi: 10.1259/bjr/69471744

Embade, N., Mariño, Z., Diercks, T., Cano, A., Lens, S., Cabrera, D., et al. (2016). Metabolic characterization of advanced liver fibrosis in HCV patients as studied by serum 1H-NMR spectroscopy. PLoS ONE 11:e0155094. doi: 10.1371 /journal.pone.0155094

Fuchs, A., and Colonna, M. (2011). Natural killer (NK) and NK-like cells at mucosal epithelia: mediators of anti-microbial defense and maintenance of tissue integrity. Eur. J. Microbiol. Immunol. 1, 257-266. doi: 10.1556/EuJMI.1.2011.4.1

Hui, J. Y., Yang, M. K., Cho, D. H., Li, A., Loke, T. K., Chan, J. C.,et al. (2007). Pyogenic liver abscesses caused by Klebsiella pneumoniae: US appearance and aspiration findings. Radiology 242, 769-776. doi: 10.1148/radiol.2423051344

Keynan, Y., Karlowsky, J. A., Walus, T., and Rubinstein, E. (2007). Pyogenic liver abscess caused by hypermucoviscous Klebsiella pneumoniae. Scand. J. Infect. Dis. 39, 828-830. doi: $10.1080 / 00365540701266763$

\section{SUPPLEMENTARY MATERIAL}

The Supplementary Material for this article can be found online at: https://www.frontiersin.org/articles/10.3389/fcimb. 2018.00174/full\#supplementary-material

Supplementary Table 1|The concentration of different metabolites in plasma in both groups and the name of metabolites involved in the pathway analysis.

Lee, N. K., Kim, S., Lee, J. W., Jeong, Y. J., Lee, S. H., Heo, J., et al (2011). CT differentiation of pyogenic liver abscesses caused by Klebsiella pneumoniae vs. non-Klebsiella pneumoniae. Br. J. Radiol. 84, 518-525. doi: $10.1259 /$ bjr $/ 23004588$

Li, Z., Du, B., Li, J., Zhang, J., Zheng, X., Jia, H., et al. (2017). Cerebrospinal fluid metabolomic profiling in tuberculous and viral meningitis: screening potential markers for differential diagnosis. Clin. Chim. Acta. 466, 38-45. doi: 10.1016/j.cca.2017.01.002

Liao, W. I., Tsai, S. H., Yu, C. Y., Huang, G. S., Lin, Y. Y., Hsu, C. W., et al. (2012). Pyogenic liver abscess treated by percutaneous catheter drainage: MDCT measurement for treatment outcome. Eur. J. Radiol. 81, 609-615. doi: 10.1016/j.ejrad.2011.01.036

Lin, J. C., Chang, F. Y., Fung, C. P., Xu, J. Z., Cheng, H. P., Wang, J. J., et al. (2004). High prevalence of phagocytic-resistant capsular serotypes of Klebsiella pneumoniae in liver abscess. Microbes Infect. 6, 1191-1198. doi: 10.1016/j.micinf.2004.06.003

Liu, C. H., Gervais, D. A., Hahn, P. F., Arellano, R. S., Uppot, R. N., and Mueller, P. R. (2009). Percutaneous hepatic abscess drainage: do multiple abscesses or multiloculated abscesses preclude drainage or affect outcome? J. Vasc. Interv. Radiol. 20, 1059-1065. doi: 10.1016/j.jvir.2009.04.062

Lo, J. Z., Leow, J. J., Ng, P. L., Lee, H. Q., Mohd Noor, N. A., Low, J. K., et al. (2015) Predictors of therapy failure in a series of 741 adult pyogenic liver abscesses. J. Hepatobiliary. Pancreat. Sci. 22, 156-165. doi: 10.1002/jhbp.174

Luo, Y., Wang, Y., Ye, L., and Yang, J. (2014). Molecular epidemiology and virulence factors of pyogenic liver abscess causing Klebsiella pneumoniae in China. Clin. Microbiol. Infect. 20, O818-O824. doi: 10.1111/1469-0691. 12664

Qu, T. T., Zhou, J. C., Jiang, Y., Shi, K. R., Li, B., Shen, P., et al. (2015). Clinical and microbiological characteristics of Klebsiella pneumoniae liver abscess in East China. BMC Infect. Dis. 15:161. doi: 10.1186/s12879-015-0899-7

Siu, L. K., Yeh, K. M., Lin, J. C., Fung, C. P., and Chang, F. Y. (2012). Klebsiella pneumoniae liver abscess: a new invasive syndrome. Lancet Infect. Dis. 12, 881-887. doi: 10.1016/S1473-3099(12)70205-0

Song, Q. H., Xu, R. M., Zhang, Q. H., Shen, G. Q., Ma, M., Zhao, X. P. et al. (2015). Glutamine supplementation and immune function during heavy load training. Int. J. Clin. Pharmacol. Ther. 53, 372-376. doi: 10.5414/ CP202227

Tan, L., Zhou, H. J., Hartman, M., Ganpathi, I. S., Madhavan, K., and Chang, S. (2013). Laparoscopic drainage of cryptogenic liver abscess. Surg. Endosc. 27, 3308-3314. doi: 10.1007/s00464-013-2910-y

Tredwell, G. D., Behrends, V., Geier, F. M., Liebeke, M., and Bundy, J. G. (2011). Between-person comparison of metabolite fitting for NMR-based quantitative metabolomics. Anal. Chem. 83, 8683-8687. doi: 10.1021/ac202123k

Wang, H. H., Tsai, S. H., Yu, C. Y., Hsu, H. H., Liu, C. H., Lin, J. C., et al. (2014). The association of haemoglobin $\mathrm{A}(1) \mathrm{C}$ levels with the clinical and CT characteristics of Klebsiella pneumoniae liver abscesses in patients with diabetes mellitus. Eur. Radiol. 24, 980-989. doi: 10.1007/s00330-014-3113-1

Wang, J., Yan, Y., Xue, X., Wang, K., and Shen, D. (2013). Comparison of pyogenic liver abscesses caused by hypermucoviscous Klebsiella pneumoniae and non-Klebsiella pneumoniae pathogens in Beijing: a retrospective analysis. J. Int. Med. Res. 41, 1088-1097. doi: 10.1177/03000605134 87645

Weljie, A. M., Newton, J., Mercier, P., Carlson, E., and Slupsky, C. M. (2006). Targeted profiling: quantitative analysis of $1 \mathrm{H}$ NMR metabolomics data. Anal. Chem. 78, 4430-4442. doi: 10.1021/ac060209g 
Xia, J., Sinelnikov, I. V., Han, B., and Wishart, D. S. (2015). MetaboAnalyst 3.0making metabolomics more meaningful. Nucleic Acids Res. 43,W251-W257. doi: 10.1093/nar/gkv380

Xiao, W., Chen, P., Dong, J., Wang, R., and Luo, B. (2015). Dietary glutamine supplementation partly reverses impaired macrophage function resulting from overload training in rats. Int. J. Sport Nutr. Exerc. Metab. 25, 179-187. doi: 10.1123/ijsnem.2014-0118

Yeh, K. M., Chang, F. Y., Fung, C. P., Lin, J. C., and Siu, L. K. (2006). magA is not a specific virulence gene for Klebsiella pneumoniae strains causing liver abscess but is part of the capsular polysaccharide gene cluster of K. pneumoniae serotype K1. J. Med. Microbiol. 55(Pt 6), 803-804. doi: 10.1099/jmm.0.46368-0
Conflict of Interest Statement: The authors declare that the research was conducted in the absence of any commercial or financial relationships that could be construed as a potential conflict of interest.

Copyright (c) 2018 Chang, Wang, Li, Liu and Zheng. This is an open-access article distributed under the terms of the Creative Commons Attribution License (CC $B Y)$. The use, distribution or reproduction in other forums is permitted, provided the original author(s) and the copyright owner are credited and that the original publication in this journal is cited, in accordance with accepted academic practice. No use, distribution or reproduction is permitted which does not comply with these terms. 